\title{
Qualitative Forschung und ihre Methoden in der Erziehungswissenschaft - auf Konsolidierungskurs?
}

\author{
Sabine Reh
}

Sammelrezension zu

1. Burkhard Fuhs: Qualitative Methoden in der Erziehungswissenschaft. Darmstadt: Wissenschaftliche Buchgesellschaft 2007. 141 S. ISBN 978-3-534-17529-1. Preis: $14,90 €$.

2. Ralf Bohnsack: Qualitative Bild-und Videointerpretation. Opladen: Barbara Budrich 2009. 267 S. ISBN 978-3-8252-8407-7. Preis: 17,90€.

3. Heide von Felden (Hrsg.): Perspektiven erziehungswissenschaftlicher Biographieforschung. Wiesbaden: VS Verlag für Sozialwissenschaften 2008. 228 S. ISBN 978-3531-15611-8. Preis: 29,90€.

4. Bettina Hünersdorf/Christoph Maeder/Burkhard Müller (Hrsg.): Ethnographie und Erziehungswissenschaft. Methodologische Reflexionen und empirische Annäherungen. Weinheim: Juventa 2008. 269 S. ISBN 978-3-7799-1700-7. Preis: 23,- $€$.

Misst man es an der Existenz entsprechender Professuren, an der Präsenz qualitativer Methoden in Ausbildungsgängen und in der Fachgesellschaft, lässt sich durchaus von einer Konsolidierung qualitativer Forschung in der Erziehungswissenschaft sprechen. Publikationsmöglichkeiten, die Zahl der Veröffentlichungen und der Debatten über Qualität und Qualitätskriterien qualitativer Forschung weisen in die gleiche Richtung. Bekannte Einführungen in Auswertungsmethoden qualitativer Forschung erleben mehrere Auflagen - zu denken ist etwa an die Einführung in das Interpretationsverfahren der Objektiven Hermeneutik von Andreas Wernet oder die Einführung in die Rekonstruktive Sozialforschung von Ralf Bohnsack.

Die vorliegenden vier Bücher gestalten in unterschiedlicher Weise diesen Trend mit das eine, indem es - wie derzeit, mit der Verankerung von Bachelor- und Masterstudiengängen, nicht unüblich - eine Einführung in die qualitative erziehungswissenschaftliche

Online publiziert: 07.10 .2009

(C) VS-Verlag 2009

Univ.-Prof. Dr. S. Reh $(\bowtie)$

Institut für Erziehungswissenschaft, Allgemeine und Historische Erziehungswissenschaft

Technische Universität Berlin, Franklinstr. 28/29, 10587 Berlin, Deutschland

E-Mail: sabine.reh@tu-berlin.de 
Forschung bietet, das andere, indem es eine Einführung in die und eine Positionsbestimmung zur Bild- und Videointerpretation liefert, einem jüngst aus verschiedenen Gründen mehr und mehr in den Fokus rückendes Feld qualitativer Forschung. Beim dritten und vierten hier vorgestellten Buch handelt es sich um Reader, die theoretische und empirisch begründete methodologische Reflexionen zur Biographieforschung - eine der Wurzeln der qualitativen Forschung in der Erziehungswissenschaft - beziehungsweise zur Ethnographie - eine in anderen Disziplinen etablierte Art der Forschung, die derzeit immer größeres Interesse in der Erziehungswissenschaft findet - bieten.

Fuhs, Qualitative Methoden in der Erziehungswissenschaft. Das Anliegen von Burkhard Fuhs ist es, eine grundlegende Einführung in Methoden erziehungswissenschaftlichqualitativer Forschung zu geben. „Nach einer Einführung in das qualitative Denken“ und „Zugängen zu Sinnwelten“ folgen unter dem Titel „Praxis der Forschung“ Ausführungen zum Forschungsprozess, zu „Struktur, Methoden und Forschungsrichtungen“, ein Kapitel zum Sampling, zur Methodenauswahl und zur Erhebung und Auswertung von Daten am Beispiel von Interviews. Anschließend werden zwei Felder qualitativer Forschung, nämlich die Biographieforschung und die erziehungswissenschaftliche Ethnographie, beispielhaft dargestellt. Argumentationsgang und Aufbau der Einführung sind so weitgehend an der Pragmatik von Forschungsprozessen orientiert, die den ,roten Faden“ der Darstellung vorgibt. Das führt zu Einbußen im Hinblick auf eine dem Gegenstand - qualitative erziehungswissenschaftliche Forschung und ihre Methoden - angemessene Darstellung, die auch demjenigen, der noch nicht geforscht hat, Strukturierungsmöglichkeiten für eine systematische Erschließung dieses weiten Feldes bietet. Das fehlende Personenregister und die nicht durchschaubare Auswahl der Schlagworte im Sachregister erschweren Nutzungsmöglichkeiten des Buches.

Immer wieder wird betont, dass es keine einheitliche Definition gibt, keinen Konsens über Anwendungs- und Geltungsbereiche und kurzerhand wird dieses zur Stärke umdefiniert, weil in ,einer komplexen, sich rasch verändernden Welt“ (S. 13) zu schnelle Vereinfachungen nicht angemessen seien. Die Definition, qualitative Forschung arbeite theoretisch geleitet und methodisch systematisch kontrolliert an der Grenze zweier Bedeutungswelten, bestimmt das Verstehen fremder Sinnwelten zum eigentlichen Merkmal qualitativer Forschung. Gerade wenn eine solche überzeugt, schiene es sinnvoll, anschließend einen systematischen Erklärungsversuch anzubieten. Wie aber daraus erziehungswissenschaftliche Forschung wird und vor allem welche Merkmale - unter Umständen besondere Gegenstandskonstruktionen, z.B. Biographie, Bildungsprozess, pädagogische Kommunikation, Habitus, Lernkultur um nur einige zu nennen - diese charakterisieren, bleibt unklar. Von einem solchen Versuch - das wäre von einer Einführung zu erwarten entbindet der Hinweis auf Komplexität und fehlende Einheitlichkeit nicht. Eine mögliche Gliederung für die Einführung (Was ist qualitative Forschung im Gegensatz zu anderen Verfahren? Was kennzeichnet die erziehungswissenschaftliche qualitative Forschung und was sind ihre Gegenstände? Welche Forschungsfragen können gestellt werden und was sind die Gründe dafür, sich diesen mit bestimmten Methoden zu nähern? Was sind Daten in qualitativer erziehungswissenschaftlicher Forschung? Mithilfe welch unterschiedlicher Verfahren werden welche verschiedenen Formen von Daten gesammelt oder erzeugt? Mit welchen unterschiedlichen Verfahren können sie ausgewertet werden? Welche Kritik gibt 
es am qualitativen Vorgehen und wie werden bestimmte Probleme - etwa das der Verallgemeinerbarkeit bzw. das der Repräsentativität - gelöst?) böte demgegenüber systematisch Strukturierungen und Unterscheidungen zur Erschließung des Feldes qualitativer Forschung, z.B. die Darlegung einer prinzipiellen Unterscheidung zwischen im weitesten Sinne codierenden und sequentiell-rekonstruktiv vorgehenden Interpretationsverfahren. Das vom Autor gewählte beispielhafte Vorgehen führt notwendigerweise zu einigen Lücken in der Darstellung, die dann, wenn die systematische Rahmung in der oben beschriebenen Form nicht erkennbar ist, umso schwerer wiegen. So spielen wichtige und in der erziehungswissenschaftlichen Forschung oft genutzte Verfahren der Auswertung von Daten in dieser Einführung keine Rolle - weder die Dokumentarische Methode noch die Objektive Hermeneutik werden Gegenstand einer kurzen Erläuterung, die immer wichtiger werdenden Bildinterpretationsverfahren oder gar die Auswertungsverfahren für videographische Daten werden zwar kurz im Bericht über die ethnographische Forschung erwähnt, haben aber auch keinen systematischen, eigenständigen Ort. Die nachvollziehbare Entscheidung, zwei Felder qualitativer Forschung, nämlich die Biographieforschung (der zu Unrecht pauschalierend vorgeworfen wird, keinen Bezug zur Bildungstheorie zu besitzen) und die erziehungswissenschaftliche Ethnographie darzustellen, erzeugen vor dem skizzierten Hintergrund ebenfalls Probleme. Das großes Feld der qualitativen Schulund Unterrichtsforschung, in der vor allem pädagogische Kommunikationen und subjektive Aneignungsprozesse bzw. -formen (und damit nicht ganz unerhebliche Gegenstände für die erziehungswissenschaftliche Forschung) analysiert oder rekonstruiert werden, findet hier keinen Platz. Das Buch lässt daher selbst die Rezensentin ein wenig ratlos zurück: Viel wird angesprochen, aber nicht immer begrifflich präzise und verstehbar miteinander in Beziehung gesetzt. Ein wirklicher Versuch, das Feld qualitativer Forschung trotz und gerade in seiner Komplexität zu strukturieren, wird nicht vorgenommen - für eine Einführungslektüre wäre das wünschenswert. Das Buch kann leider auf diese Weise auch nicht unbedingt dazu dienen, alte Vorurteile gegen eine unsystematische qualitative Forschung aus dem Weg zu räumen.

Bohnsack, Qualitative Bild- und Videointerpretation. Ralf Bohnsack dagegen scheint es einfacher zu haben, wenn er sich dem immer wichtiger werdenden methodisch kontrollierten Zugang zum Bild widmet. Er präsentiert in klarer Form ein konsistentes Verfahren der qualitativen Forschung, nämlich eine ,dokumentarische Bild- und Videointerpretation", und entwickelt damit die bisher zumeist nur auf Texte angewandte und für diese breit beschriebene dokumentarische Methode weiter. Er charakterisiert das Anliegen seines Buches als die Darlegung einer ,in sich geschlossene[n] Methodologie und Methodik der Interpretation des stehenden und bewegten Bildes von sozialwissenschaftlicher Relevanz [...], welche der Eigenlogik des Ikonischen und dessen unterschiedlichen Gattungen im Bereich des stehenden wie des bewegten Bildes gerecht zu werden vermag“ (S. 12 f.).

Ausgehend von einer kurzen Skizze der allgemeinen Grundlagen der dokumentarischen Methode wird im zweiten Schritt das Vorgehen einer Bildinterpretation entwickelt und begründet. Bohnsack reklamiert, die vorreflexive, implizite Verständigung im Medium des Bildes und damit einen bedeutsamen und bisher in qualitativer Forschung eher vernachlässigten Teil unserer alltäglichen Verständigung untersuchbar machen zu 
wollen. Unabdingbar für die Entwicklung eines solchen Verfahrens ist ein grundlagentheoretischer Zugang zu dieser Art des atheoretischen Wissens, den Bohnsack in den Theorie- und Beschreibungsangeboten von Panowsky, seinem Entwurf einer auf der Ikonographie aufbauenden Ikonologie und deren Reinterpretation durch Max Imdahl sieht. Mit dessen Vorschlag einer Ikonik, die auf der nicht-ikonographischen (einer, wie Bohnsack sagt, denotativen Ebene) liegt, kann die formale Komposition eines Bildes analysiert, kann der Eigensinn der Bilder erkannt werden, indem die Wahrnehmung des literarischen oder szenischen Gehaltes suspendiert wird. Entsprechend und analog zu den Verfahrensschritten der dokumentarischen Textinterpretation und der Mannheimschen Unterscheidung von immanentem und dokumentarischem Sinngehalt schlägt Bohnsack vor, in der „formulierenden Interpretation“ das „Was“ des Bildes, vor-ikonographisch und ikonographisch zu beschreiben und in der, ,reflektierenden Interpretation“ die Art der Darstellung, das „Wie“, zum Gegenstand zu machen, die formale Struktur des Bildes, die „planimetrische Ganzheitsstruktur“, die ,,szenische Choreographie“ und die ,perspektivische Projektion“" zu untersuchen - und damit das Nicht-Narrative, die Simultaneität eines Bildes zu erfassen.

So überzeugend dieses Verfahren, gerade auch in den dokumentierten Interpretationsbeispielen, vorgeführt wird, so schwierig und vielleicht auch problematisch wird es bei der Video- und Filminterpretation, da hierfür bisher keine auf die visuelle Ebene abzielenden Auswertungsverfahren existieren und sich das Problem bzw. der Widerspruch von Sequenzanalysen und Analysen, die auf Simultaneität (also mit Bohnsack auf Bildhaftigkeit) zielen, zuspitzt. Bohnsack begreift den Film als ,ein Bild, das sich bewegt“ und nicht primär als ikonographische Narration. Die Nähe zum Bild, also zur Fotografie und die Besonderheit des Films sind - darauf weist Bohnsack hin - in der Filmtheorie umstritten: Während Kracauer etwa die Nähe des Films zum Foto hervorhebt, sehen andere in der Folge der vielen einzelnen Bilder das Wesentliche, mit der die Einheit und damit das Charakteristische des Films geschaffen werde: Indem Bilder als Bilderfolge erscheinen, in denen Bewegungen gezeigt werden, ist ihnen eine zeitliche und damit narrative Dimension eingeschrieben (die im Übrigen, möglicherweise gegen Bohnsack formuliert, nicht mit Sprachlichkeit verwechselt werden darf). Um Bewegungen analysieren zu können, trifft Bohnsack Unterscheidungen zwischen einem vor-ikonographischen, aufwendig und technisch anmutenden Beschreibungs-Modus und der ikonographischen Beschreibung, mit der - verstehend übrigens - Motivationsstrukturen unterstellt werden. Warum Bohnsack hier auf den Handlungsbegriff von Habermas zurückgreift, bleibt unklar, entspräche doch der Begriff der Praktiken, deren teleoaffektive Struktur eingelagertes Wissen enthält und die immer in Simultaneität erfassender Wahrnehmung verstanden werden, seiner praxeologischen Einstellung. Das Vorgehen in der Videoanalyse, das Bohnsack anschließend unterbreitet, mutet dann nicht nur kompliziert an, sondern lässt offen, ob es einen Ort für die Erfassung der Narrativität des Films - die in der Verdoppelung von diegetischer und mimetischer Narration die spezifische Qualität des Films erzeugt - gibt.

Die beiden Sammelbände von Heide von Felden sowie von Hünersdorf, Maeder und Müller sind nicht ausschließlich methodologischen und methodischen Fragen gewidmet. Vielmehr wollen sie einen Stand der Diskussion eines etablierten Feldes erziehungswissenschaftlicher Forschung, der Biographieforschung, darstellen bzw. diskutieren die 
Bedeutung einer in anderen Disziplinen etablierten Forschungsrichtung für die Erziehungswissenschaft: Was kann ethnographische Forschung zur Erschließung des erziehungswissenschaftlichen Gegenstandes beitragen?

Von Felden (Hrsg.), Perspektiven erziehungswissenschaftlicher Biographieforschung. Heide von Felden referiert als Herausgeberin den Entwicklungsstand methodischer Diskussionen in der Biographieforschung und rezipiert gegenwärtige Diskussionen als „,[m]ethodische Differenzierungen“. Der Teil des Buches, der mit vier Aufsätzen methodischen Fragen gewidmet ist - und der allein hier betrachtet wird - soll dieses dokumentieren. Während der Beitrag von Seltrecht nun eigentlich solche Fragen nicht berührt und falsch eingeordnet erscheint, diskutieren Bartmann und Kunze zentrale methodische Probleme der an Schützes Verfahren orientierten Biographieforschung. Sie zeigen auf, dass in narrativen Interviews auch Textteile, die Argumentationen enthalten bzw. darstellen - anders als Schütze proklamiert - einen Zugang zur Erfahrungsebene bieten, etwa indem anschließend detailliert erzählt wird. Keinesfalls stellen sie einen defizienten Biographisierungsmodus dar, sondern können Ausdruck von Ressourcen, biographischer Reflexivität und Fähigkeit zum Perspektivenwechsel sein. Das stellen die Autorinnen überzeugend dar, bestätigen aber deutlich auch zwei grundlegende und immer wieder diskutierte Probleme des Ansatzes von Schütze: Die Textsortenbestimmung ist oft nicht trennscharf und die Tatsache, dass auch Erzählungen aus gegenwärtiger Sicht vorgetragen werden, wird immer wieder übersehen. In den anderen beiden Beiträgen wird einmal dafür plädiert, eine Biographieforschung zu etablieren, die sich für die Abhängigkeit biographischer Erzählungen von Erziehungs-, Bildungs- und Lernprozessen interessiert. Diese hätte - ein solches Projekt wird angekündigt - echte Längsschnittstudien zu entwerfen und durchzuführen (Kade \& Hof). In einem weiteren Aufsatz geht Anne Schlüter mithilfe theoretischer und methodischer Annahmen der Frage nach, welche Bedeutung die Eingangssequenz eines narrativen Interviews für dessen gesamte Auswertung hat. Sie kommt zu der allerdings nicht neuen Auffassung, dass hier schon entscheidende Strukturelemente - die Sicht auf das Ich, die Perspektivität u.a.m. - gelegt bzw. erkennbar werden. Vorsichtig ließe sich aus dem methodischen Teil dieses Bandes ein Trend formulieren: Gegenwärtig bemüht sich die Biographieforschung darum, die Veränderlichkeit autobiographischer Erzählungen und damit die Gegenwärtigkeit des Erzählens auch methodisch einholen zu können.

\section{Hünersdorf, Maeder, Müller (Hrsg.), Ethnographie und Erziehungswissenschaft.} Auch dieser Band hat einen Teil seiner Aufsätze methodologischen und methodischen Fragen gewidmet. In einem interessanten Aufsatz, der zwar nicht in den methodologischen Teil eingeordnet ist, schlagen Bollig und Kelle auf der Suche nach einer ethnographischen Antwort auf die erziehungswissenschaftliche Frage nach der Formbestimmung der Pädagogik vor, von einem abstrakten Feldbegriff auszugehen, der sich nicht an Teilnehmerkonzeptionen orientiert. Kennzeichen des pädagogischen Feldes sei die Tatsache, dass Personen ein Defizit zugeschrieben werde, das durch Lernen veränderbar erscheine. Der Blick kann sodann auf unterschiedliche Praktiken gerichtet werden, in denen eine solche Adressierung möglich erscheint. Hybride Praktiken erlauben es, pädagogische Interaktionen als eingelagert in Anderes, z.B. in ihrer medizinischen Logik und Funktionalität, 
in ihrer Komplexität zu erkennen und einen Beitrag zur Frage nach der Formbestimmung des Pädagogischen aus praxeologischer Forschung heraus zu leisten. Damit zeigt dieser Beitrag einen Weg, die dem Band vorangestellte Frage nach den Leistungen der ethnographischen Forschung für die Erziehungswissenschaft zu beantworten. Weitere methodologische Beiträge stellen zum einen heraus, was das Spezifische der Ethnographie des pädagogischen Feldes ist, und zeigen gegenwärtige Trends auf. Cloos beschäftigt sich mit der Frage nach der „Mehrfachrolle“, auch einer pädagogischen des ethnographischen Forschers im Feld, die damit die ethnographische Forschung hier in besonderer Weise ausgesprochen komplex gestalte. In pädagogischen Feldern finde sich eine Art ethnographisch zu forschen, die thematisch und zeitlich stark fokussiert ist. Deren Konzept und Stellenwert diskutiert Oester in einem Beitrag. Wagner-Willi stellt die - an Bohnsacks Verfahren orientierte - dokumentarische Videointerpretation in der ethnographischen Forschung vor. Troman und Jeffrey diskutieren Möglichkeiten, Methodologie und Methode international vergleichender erziehungswissenschaftlicher Ethnographie, wie wir sie gegenwärtig zunehmend in ethnographisch ausgerichteten Forschungen jüngerer Wissenschaftlerinnen finden.

Die vorgestellten Bücher dokumentieren zwar Schwierigkeiten einer zusammenfassenden, systematisierenden Darstellung des weiten Feldes qualitativer Methoden in der Erziehungswissenschaft, aber gleichzeitig auch die Konsolidierung dieses Forschungsfeldes als Ausdifferenzierung unterschiedlicher Vorgehensweisen und der Entwicklung neuer Erhebungs- und Auswertungsverfahren. 